\title{
Random Walks with Look-ahead in Scale-free Random Graphs
}

\author{
Colin Cooper* $\quad$ Alan Frieze ${ }^{\dagger}$
}

March 20, 2010

\begin{abstract}
If $m \geq 2$ is constant and $0 \leq r \leq \varepsilon \log \log n$ for a small positive constant $\varepsilon$, then whp a random walk with look-ahead $r$ on a scale-free graph $\left.G=G_{(} m, n\right)$ has cover time $C_{G}(r) \sim\left(2 /\left(m^{r-1}(m-1)\right)\right) n \log n$.
\end{abstract}

\section{Introduction}

Random graphs with degree sequences exhibiting power law properties have been studied by many authors and in many contexts. Recent interest was stimulated by the papers of Barabási and Albert [1] who observed a power law degree sequence for a subgraph of the World Wide Web (www) and by Faloutsos, Faloutsos and Faloutsos [10] who observed power law behaviour for the internet graph. Empirical studies giving power laws for a larger portion of the WWW were subsequently made by other researchers, in particular Broder et al. [3].

An established method of searching large or unknown networks, is to use a random walk. Let $G=(V, E)$ be a connected graph with $|V|=n$ vertices. For $v \in V$ let $C_{v}$ be the expected time taken for a simple random walk $W$ on $G$ starting at $v$, to visit every vertex of $G$. The vertex cover time $C_{G}$ of $G$ is defined as $C_{G}=\max _{v \in V} C_{v}$.

Modifying random walks to reduce the cover time, $C_{G}$, is a matter of considerable practical and theoretical interest. One possible approach is to use look-ahead (probing to fixed depth). A random walk has look-ahead $r$, if at each step the walk explores all neighbours of the current vertex to depth $r$. More formally, for integer $r \geq 0$ and $v \in V$ we let $C_{v}(r)$ be the expected

\footnotetext{
*Department of Computer Science, King's College, University of London, London WC2R 2LS, UK

†Department of Mathematical Sciences, Carnegie Mellon University, Pittsburgh PA15213. Supported in part by NSF grant CCR-0200945.
} 
time for a simple random walk starting at $v$ to pass within distance $r$ of every vertex of $G$. Then we let $C_{G}(r)=\max _{v \in V} C_{v}(r)$. Thus look-ahead $C_{G}(0)=C_{G}$.

In the case of random regular graphs, the performance of look-ahead $r$ walks is given by the following theorem of [6].

Theorem 1. Let $C_{G}(r)$ be the expected number of steps needed for a random walk to get within distance $r$ of every vertex of a graph $G$. Let $d \geq 3, r \geq 0$ be constants. Let $\mathcal{G}_{d}$ denote the set of $d$-regular graphs with vertex set $V=\{1,2, \ldots, n\}$. If $G$ is chosen randomly from $\mathcal{G}_{d}$, then whp

$$
C_{G}(r) \sim \frac{1}{(d-2)(d-1)^{r-1}} n \log n .
$$

In this paper we study look-ahead random walks in scale-free random graphs $G(m, n)$. The precise definition of $G(m, n)$ is given in Section 2 below. The cover time of scale-free graphs $G(m, n)$, established in [7], is $C_{G} \sim \frac{2 m}{(m-1)} n \log n$. We generalize that result as follows.

Theorem 2. If $m \geq 2$ constant, and $0 \leq r \leq \varepsilon \log \log n$ for a positive constant $\varepsilon<1 / \log m$, then whp a random walk with look-ahead $r$ on a scale-free graph $G=G(m, n)$ has cover time

$$
C_{G}(r) \sim \frac{2}{m^{r-1}(m-1)} n \log n .
$$

There are several distinct methods to generate a random graph with a given power law degree sequence. One method is to use a process model, in which new vertices join using preferential attachment; e.g. the scale-free model $G(m, n)$ of [2] used in this paper. Another method is to use a configuration model to generate a random graph with a fixed degree sequence $\boldsymbol{d}$ of a power law type, or expected degree sequence $\overline{\boldsymbol{d}}$ of a power law type. In Random walks with look-ahead in power law random graphs [13], Mihail, Sabieri and Tetali study lookahead random walks on random graphs $G(\overline{\boldsymbol{d}})$ generated by a configuration model, in which $\operatorname{Pr}(d(v)=k)=c / k^{2+\epsilon}$ for $d_{\min } \leq k \leq \sqrt{n}$. They find that:

Theorem 3. [13] For any $\delta, 0<\delta<1 / 2$, the expected number of steps for a random walk with look-ahead 1 to discover $\Omega\left(n^{1-\epsilon(1 / 2-\delta)}\right)$ vertices is $O\left(n^{1 / 2+\delta}\right) \log n$ whp.

For any $\delta, 0<\delta<1 / 2$, the expected number of steps for a random walk with look-ahead 2 to discover $\Omega\left(n^{1-2 \epsilon(1 / 2-\delta)-\delta}\right)$ vertices is $O\left(n^{\epsilon(1 / 2-\delta)}\right) \log n$ whp.

The theorem shows the rapid (super-linear) initial rate of discovery of vertices using lookahead. Many of the vertices discovered quickly by the walk are neighbours of the $\Omega\left(n^{1 / 2-\epsilon(1 / 2-\delta)+\delta}\right)$ vertices of degree $\geq n^{1 / 2-\delta}$ (large vertices). The precise argument for lookahead 2, hinges on the fact that each large vertex has $\Omega\left(n^{1 / 2-\delta}\right)$ neighbours of degree $d_{\text {min }}$, and that each large neighbour has $\Omega\left(n^{1 / 2-2 \epsilon(1 / 2-\delta)}\right)$ other large neighbours.

In process models using preferential attachment, the edge density between the large vertices is much less, and it is not apparent that the results of Theorem 3 still hold. For example, if we 
use the web-graph model of [5], [4] to generate a graph with power law $2+\epsilon$, then, whp, the number of edges between large vertices is $\tilde{O}\left(n^{1 / 2+\delta-\epsilon(1 / 2-\delta)}\right)$. This differs from the model used in Theorem 3, where the expected number of edges between large vertices is $\Theta\left(n^{1-2 \epsilon(1 / 2-\delta)}\right)$. The edge density between large vertices in the web-graph model, can be deduced as follows: At step $n$, whp the degree of a vertex added at step $s$ is $\tilde{O}(n / s)^{1 /(1+\epsilon)}$. For this to be at least $n^{1 / 2-\delta}$, we need $s=\tilde{O}\left(n^{1 / 2+\delta-\epsilon(1 / 2-\delta)}\right)$.

\section{Scale-free graphs $G(m, n)$}

A common method to generate random graphs with a power law degree sequence, is to use a preferential attachment process. Many variants of the process method exist. We use the scale-free model of [2]. In this model, a graph $G(1, m n)$ is generated sequentially as follows. At step $t=0$ the graph is empty, and $V(0)=\emptyset$. Let $\delta_{t}(v)$ denote the degree of vertex $v \in V(t)$ at the end of step $t$. At each step $t=1,2, \ldots, n$, add vertex $v_{t}$ to $V(t-1)$, and an edge from $v_{t}$ to a vertex $u$, chosen at random from existing vertices according to the distribution:

$$
\operatorname{Pr}\left(u=v_{i}\right)= \begin{cases}\frac{\delta_{t-1}\left(v_{i}\right)}{2 t-1}, & \text { if } v_{i} \neq v_{t} \\ \frac{1}{2 t-1}, & \text { if } v_{i}=v_{t}\end{cases}
$$

The graph $G(m, n)$ is formed from $G(1, m n)$ as follows. Every $m$ steps contract the most recently added $m$ vertices $v_{m(k-1)+1}, \ldots, v_{m k}$ to form a single vertex $k=1,2, \ldots$. Let $G(m, n)$ denote the random graph formed from $G(1, m n)$ at time step $m n$ after $n$ contractions of size $m$. Thus $G(m, n)$ has $n$ vertices and $m n$ edges and may be a multi-graph. It can be thought of as the final graph in a sequence $G(m, 1), G(m, 2), \ldots, G(m, n)$. The degree of a vertex $v$ in $G(m, n)$ is denoted by $d_{n}(v)$.

\subsection{Properties of nice scale-free graphs}

Let $0<\epsilon<1 / \log m$ and let

$$
\omega=(\log n)^{1 / 3}, \quad \omega_{0}=\epsilon \log \log n .
$$

For $v \in V$ and $l \geq 0$, let $N_{l}^{G}(v)=N_{l}(v)=\{w: \operatorname{dist}(v, w)=l\}$ be the set of vertices at distance $l$ from $v$. Let $M_{r}^{G}(v)=M_{r}(v)$ be the subgraph of $G$ induced by $\cup_{l=0}^{r} N_{l}(v)$.

- A vertex $v$ is locally tree-like to depth $k$ if the sub-graph $M_{k}(v)$ is a tree. A vertex $v$ is locally tree-like if it is locally tree-like to depth $2 \omega$.

- A vertex $v$ is locally regular, if it has $d(v)=d_{n}(v)=m$, is locally tree-like and the vertices at distance $2 \omega_{0}$ or less have branching factor $m$. 
- A cycle $C$ is small if $|C| \leq 2 \omega+1$. Thus a locally-tree-like vertex is distance at least $2 \omega$ from any small cycle.

- A vertex $v$ is light if $v>n^{1 / 10}$ and heavy otherwise. A cycle is light if it only contains light vertices.

A graph $G(m, n)$ is nice if it has the following properties: The references in items 1.-7. indicate where it is shown that $G(m, n)$ has these properties whp. We will have to prove here that 8 . holds whp.

P1 Maximum vertex degree (Lemma 6 of [7]):

$$
\nexists(k, \ell), 1 \leq k \leq \ell \leq n: d_{\ell}(k) \geq(\ell / k)^{1 / 2} \log ^{3} n \text { and } \nexists v \leq n^{1 / 10}: d_{n}(v) \leq n^{1 / 4} .
$$

P2 There are $n^{1-o(1)}$ locally regular vertices $v$ with $M_{2 \omega_{0}}(v) \subseteq[n / 2, n]$.

(Lemma 12 of [7]. The proof there uses $\omega_{0}=\log \log \log n$ but $\omega_{0}=\epsilon \log \log n$ will suffice. To be precise, in the last two paragraphs of that proof, we require that $n 2^{-10 \mathrm{~km} k}=n^{1-o(1)}$ which it is when $k=\omega_{0}$ and $\epsilon \log m<1$ ).

P3 No small cycle is within distance $10 \omega$ of a distinct light cycle. (Corollary 8 of [7]).

P4 There are at most $(\log n)^{10 \omega}$ vertices on small cycles. (Lemma 9 of [7]).

P5 There are at most $n /(\log n)^{\omega}$ vertices $v \geq n / 2$ which have more than $(\log n)^{11 \omega}$ vertices at distance $3 \omega$ or less from them.

P6 There are $O\left(n^{1 / 2+o(1)}\right)$ non locally tree-like vertices. (Lemma 10 of [7]).

P7 $G(m, n)$ has conductance $\Phi$ bounded away from zero [12].

P8

$$
\nexists v \leq n^{1 / 10} \text { such that } \sum_{x \in M_{2 \omega_{0}}(v)} d(x) \geq n^{9 / 10} .
$$

Lemma 4. For $m \geq 2, G(m, n)$ is nice whp.

Proof We only need to prove item 8. This uses

$$
\left.\operatorname{Pr}(G(m, n) \text { contains edge }(\alpha, \beta)) \mid d_{\beta}(\alpha) \leq(\beta / \alpha)^{1 / 2}(\log n)^{3}\right) \leq \frac{\log ^{3} n}{(\alpha \beta)^{1 / 2}} .
$$

Furthermore, this remains an upper bound if we condition on the existence of some of the other edges in $G(m, n)$. This is equation (30) of [7]. 
Fix $v \leq n^{1 / 10}$. Let $Z_{v}$ be the number of paths of length at most $2 \omega_{0}$ with $v$ as an endpoint. Then

$$
\mathbf{E}\left(Z_{v}\right) \leq \sum_{k=1}^{2 \omega_{0}} \sum_{v_{1}, \ldots, v_{k}} \frac{\log ^{3 k+3} n}{v^{1 / 2} v_{k}^{1 / 2}} \prod_{i=1}^{k-1} \frac{1}{v_{i}} \leq \frac{1}{v^{1 / 2}} \sum_{k=1}^{2 \omega_{0}} \log ^{3 k+3} n H_{n}^{k-1} \sum_{v_{k}} \frac{1}{v_{k}^{1 / 2}} \leq n^{1 / 2+o(1)} .
$$

So

$$
\operatorname{Pr}\left(\exists v \leq n^{1 / 10}: Z_{v} \geq n^{3 / 4}\right) \leq n^{1 / 10} n^{-1 / 4+o(1)}=o(1)
$$

Thus whp $\left|M_{2 \omega_{0}}(v)\right| \leq n^{3 / 4}$ for $v \leq n^{1 / 10}$. Applying (3) we see that whp

$$
\sum_{x \in M_{2 \omega_{0}}(v)} d(x) \leq n^{1 / 2} \log ^{3} n \sum_{k=1}^{n^{3 / 4}} k^{-1 / 2} \leq n^{7 / 8+o(1)}
$$

\section{First visit time lemma}

For a random walk $\mathcal{W}_{v}^{G}=\mathcal{W}_{v}$, starting at $v$, let

$$
P_{v}^{(t)}(x)=\operatorname{Pr}\left(\mathcal{W}_{v}(t)=x\right)
$$

and

$$
r_{t}=r_{t}(v, G)=P_{v}^{(t)}(v)
$$

be the probability that the walk returns to $v$ at step $t=0,1, \ldots$ In particular note that $r_{0}=1$ as the walk starts on $v$.

Let $\pi_{v}=\frac{d_{n}(v)}{2 m}$ denote the steady state distribution of the random walk $\mathcal{W}_{v}$ for all $v \in V$.

Let

$$
R(z)=\sum_{t=0}^{\infty} r_{t} z^{t}
$$

generate $r_{t}$, and let

$$
R_{T}(z)=\sum_{j=0}^{T-1} r_{j} z^{j}
$$

Thus, evaluating $R_{T}(z)$ at $z=1$, we have $R_{T}(1) \geq r_{0}=1$.

For proof of the following lemma, see [6], [8]. The lemma should be viewed in the context that $G$ is an $n$ vertex graph which is part of a sequence of graphs with $n$ growing to infinity. 
Lemma 5. Let $T$ satisfy $\max _{u, x \in V}\left|P_{u}^{(T)}(x)-\pi_{x}\right| \leq n^{-3}$, and let $R_{v}=R_{T}(1)$ where $R_{T}(z)$ is given by (5). For sufficiently large constant $K$, let

$$
\lambda=\frac{1}{K T} .
$$

Suppose also that the following conditions hold:

(a) For some constant $\theta>0$, we have

$$
\min _{|z| \leq 1+\lambda}\left|R_{T}(z)\right| \geq \theta
$$

(b) $T \pi_{v}=o(1)$ and $T \pi_{v}=\Omega\left(n^{-2}\right)$.

For $t \geq T$ let $\boldsymbol{A}_{t}(v)$ be the event that $\mathcal{W}_{u}$ does not visit $v$ at steps $T, T+1, \ldots, t$.

Then there exists

$$
p_{v}=\frac{\pi_{v}}{R_{v}\left(1+O\left(T \pi_{v}\right)\right)}
$$

such that for all $T \leq t$

$$
\operatorname{Pr}\left(\boldsymbol{A}_{t}(v)\right)=\frac{\left(1+O\left(T \pi_{v}\right)\right)}{\left(1+p_{v}\right)^{t}}+O\left(T^{2} \pi_{v} e^{-\lambda t / 2}\right) .
$$

We should perhaps warn the reader that we will be applying Lemma 5 to a graph obtained by contracting vertices within distance $r$ of some fixed vertex $v$.

\section{Random walks on scale-free graphs}

\subsection{Mixing time of walks on $G(m, n)$}

The conductance $\Phi(G)$ of a graph $G$ is defined by

$$
\Phi(G)=\min _{\pi(S) \leq 1 / 2} \frac{e(S: \bar{S})}{d(S)}
$$

where $e(S: \bar{S})$ denotes the number of edges between $S$ and $\bar{S}$ and $d(S)=\sum_{v \in S} d(v)$.

It follows from e.g. Sinclair [14] that

$$
\left|P_{u}^{(t)}(x)-\pi_{x}\right| \leq(d(x) / d(u))^{1 / 2}\left(1-\Phi^{2} / 2\right)^{t} .
$$

Let $T$ be such that, for $t \geq T$

$$
\max _{u, x \in V}\left|P_{u}^{(t)}(x)-\pi_{x}\right| \leq n^{-3}
$$


Mihail, Papadimitriou and Saberi [12] proved that the conductance $\Phi(G(m, n))$ of a simple random walk on $G(m, n)$ is bounded below by an absolute constant $\Phi>0$ (whp). We will form a graph $\Gamma$ by contracting some set of vertices of $G(m, n)$ to single vertex $\gamma$. This contraction can only increase conductance, i.e. for nice graphs $\Phi(\Gamma) \geq \Phi(G(m, n))>\Phi$. As $d(\gamma)<m n=2|E(G)|$, then condition (10) of Lemma 5 holds in both $G$ and $\Gamma$ provided we choose a mixing time

$$
T=A \log n
$$

for some large constant $A$.

We remark that there is a technical point in using (9). The result of [14] assumes that the walk is lazy, and only moves to a neighbour with probability $1 / 2$ at any step, which halves the conductance and doubles the cover time; but (asymptotically) at half the steps the particle does not move. Asymptotically the values $R_{v}$ are doubled too. Overall, the presence of loops has a negligible effect on the analysis and we will ignore this for the rest of the paper and continue as though there are no lazy steps.

\subsection{Background material on random walks}

We note some standard results. Let $v$ be a vertex of a graph $G$ and $B$ a set of vertices disjoint from $v$. The escape probability, $p_{\text {esc }}(v, B, G)$, is the probability that starting at $v$, the walk reaches $B$ before returning to $v$.

We note a property of random walks on undirected graphs (see e.g. Doyle and Snell [9]). For an unbiased random walk,

$$
p_{e s c}(v, B, G)=\frac{1}{d(v) R_{E F F}},
$$

where $R_{E F F}=R_{E F F}(v, B, G)$ is the effective resistance between $v$ and $B$ in $G$. We assume each edge of $G$ has resistance 1.

In the context of electrical networks, deleting an edge corresponds to increasing the resistance of that edge to infinity (i.e. allocating zero flow). By Raleigh's Monotonicity Law, if edges are deleted from $G$ to form a sub-graph $G^{\prime}$ then $R_{E F F}\left(v, B, G^{\prime}\right) \geq R_{E F F}(v, B, G)$. Provided we do not delete edges incident with $v$, it follows that $p_{e s c}\left(v, B, G^{\prime}\right) \leq p_{e s c}(v, B, G)$. However $p_{\text {esc }}(v, B, G)=1-\rho$, where $\rho$ is the probability that the walk returns to $v$ before absorption at $B$, and hence $\rho^{\prime} \geq \rho$. Thus $R_{v, B}$, the expected number of returns to $v$ before absorption at $B$ satisfies

$$
R_{v, B}=\frac{1}{1-\rho} \leq \frac{1}{1-\rho^{\prime}}=R_{v, B}^{\prime} .
$$

Another result we require, is the absorption probabilities for a biased random walk on the path $(0,1, \ldots, k)$ with absorbing states $0, k$. Let the transition probabilities at vertices $(1, \ldots, k-1)$ be $q=\operatorname{Pr}$ (move left), $p=\operatorname{Pr}$ (move right). Then (see e.g. Feller [11]) provided $p \neq q$, for a 
particle starting at position $i$,

$$
\operatorname{Pr}(\text { absorption at } k)=\frac{(q / p)^{i}-1}{(q / p)^{k}-1} .
$$

For the special case of a walk on the semi-infinite path $(0,1, \ldots)$, with $q<p$, and starting position $i=1$, we have

$$
\operatorname{Pr}(\text { absorption at } 0)=\frac{q}{p}
$$

\subsection{Estimating returns for look-ahead random walks}

Construction of $\gamma(v)$. To analyze the look-ahead random walk we contract $M_{r}(v)$ to a single vertex $\gamma(v)$, and delete any loops created with the exception of those arising from edges between vertices in $N_{r}(v)$. This contraction gives a graph $\Gamma=\Gamma(v, r)$. For a random walk on $\Gamma$ starting from $\gamma(v)$, let $R_{\gamma(v)}$ be the expected number of returns to $\gamma(v)$ during the mixing time $T_{\Gamma}$ i.e. a suitable value for $T$ in (11) as applied to $\Gamma$. The aim of this section is to establish bounds on the value of $R_{\gamma(v)}$.

When we contract $M_{r}^{G}(v)$ to $\gamma(v)$, the subgraph $M_{\omega+r}^{G}(v)$ of $G$ is replaced by a subgraph $M_{\omega}^{\Gamma}(\gamma(v))$ of depth $\omega$ in $\Gamma$. Let $d(\gamma(v))$ be the degree of $\gamma(v)$ in $\Gamma$. If $v$ is locally tree-like, then

$$
d(\gamma(v)) \geq d(v) m^{r}
$$

The total degree $d(\Gamma)$ of $\Gamma(v, r)$ is

$$
d(\Gamma)=2|E(G)|-d\left(M_{r}^{G}(v)\right)+2 L
$$

where $L<d\left(M_{r}^{G}(v)\right)$ is the number of edges induced by $N_{r}(v)$.

Construction of tree $\mathcal{T}$. We describe next how we now delete some edges and vertices from $M_{\omega}^{\Gamma}(\gamma(v))$ and then extend it to an infinite tree. At the $t$-th step of the construction of $G(m, n)$, the newly added vertex $v_{t}$ directs $m$ edges outward. Thus the edges of $G$ and $\Gamma$ have an underlying orientation. We let $N_{\leq \ell}^{H+}(x)$ be those vertices in $M_{\omega}^{H}(x), H=G, \Gamma$ that are reachable from vertex $x$ by a directed path of length at most $\ell$.

We regard $\gamma(v)$ as having out-degree $d^{+}(\gamma)=k$ and in-degree $d^{-}(\gamma)=l$ where $k+l=d(\gamma(v))$. If vertex $v$ is locally tree-like up to depth $\omega+r$, (or any cycles close to $v$ are contained in $\left.M_{r-1}^{G}(v)\right)$, then $\gamma(v)$ is not incident to any loops or parallel edges. In this case each out-edge $e=(\gamma, u)$ of $\gamma$ gives rise to an out-branching $T_{u}$ rooted at $u$ which has vertex set $\left.N_{\leq \omega-1}^{\Gamma+}(u)\right)$ and branching factor $m$. Now delete any exterior in-edges pointing to $T_{u}$, i.e. edges $(\bar{w}, x) \neq e$ where $x \in T_{u}$ and $w \notin T_{u}$. If $d^{-}(\gamma)>0$, then each in-edge $e=(u, \gamma)$ also gives rise to an out-branching $T_{u}$ rooted at $u$. Starting with the out-branching $T_{u}^{\prime}$ on $\left.N_{\leq \omega-1}^{\Gamma+}(u)\right)$ we delete edge $e$ from $T_{u}^{\prime}$ along with the sub-tree containing $\gamma$. This defines $T_{u}$ in this case. $T_{u}$ has out-branching factor $m-1$ at $u$ and out-branching factor $m$ at each non-leaf. As before, prune all exterior in-edges pointing to $T_{u}$ coming from outside $T_{u}$. 
Suppose that, after the deletions described above, $\gamma$ is not locally tree-like to depth $\omega$. Let $C_{1}, C_{2}, \ldots, C_{k}$ be the cycles contained in $M_{\omega}^{\Gamma}(\gamma(v))$. They are necessarily small. Using property P3 we see that there are two cases to consider. In the first case $C_{1}$ is light and $k=1$. Let $e=(x, y)$ be the edge of $C_{1}$ that is furthest from $\gamma(v)$. Break ties arbitrarily. We assume that its orientation is $x$ to $y$. We delete $e$ and prune away as in the tree-like case. If $k \geq 2$ then every cycle contains a heavy vertex. For each cycle $C_{i}$ choose an edge $e_{i}$ containing a heavy vertex $v_{i}$ and delete the edge $e_{i}$. Vertex $v_{i}$ will become an absorbing vertex for the walk.

It will be convenient for some calculations to extend $M_{\omega}(\gamma)$ outward to infinity from nonabsorbing vertices at depth $\omega$ from $\gamma$ as a regular tree with branching factor $m$. (When $m=2$ it is possible to create a single leaf $x$ at depth less than $\omega$ ). This forms an infinite tree $\mathcal{T}$ rooted at $\gamma(v)$.

Our first step is to calculate the number of returns to $\gamma$ in $\mathcal{T}$.

Lemma 6. Let $k=d^{+}(\gamma(v)), l=d^{-}(\gamma(v))$. For a random walk in $\mathcal{T}$, starting from $\gamma(v)$, let $\rho=\rho_{v}(k, l)$ be the probability of a first return to $\gamma(v)$.

(a) If $\gamma(v)$ is is locally tree-like then

$$
\rho(k, l)=\frac{k}{k+l}\left(\frac{1}{m}\right)+\frac{l}{k+l}\left(\frac{m}{m^{2}-m+1}\right) .
$$

(b) If $\gamma(v)$ is locally regular then $\rho=1 / \mathrm{m}$.

(c) If $M_{\omega}(\gamma)$ is not tree-like but contains only heavy cycles then

$$
\rho(k, l) \leq \frac{k}{k+l}\left(\frac{1}{m}\right)+\frac{l}{k+l}\left(\frac{m}{m^{2}-m+1}\right) .
$$

(d) If $M_{\omega}(\gamma)$ contains a unique light cycle $C$ then

$$
\rho(k, l) \leq \frac{k-\delta_{k}}{k+l}\left(\frac{1}{m}\right)+\frac{l-\delta_{l}}{k+l}\left(\frac{m}{m^{2}-m+1}\right)+\frac{1}{k+l}
$$

where $\delta_{k}, \delta_{l}=0 / 1$ and $\delta_{k}+\delta_{l} \leq 1$.

Proof Case (a), (b): Let

$$
\rho=\frac{k}{k+l} \rho^{+}+\frac{l}{k+l} \rho^{-}
$$

where $\rho^{+}$(respectively $\rho^{-}$) is the return probability, conditional on the walk following an edge oriented away from (respectively towards) $\gamma(v)$.

Choosing $p=m /(m+1), q=1 /(m+1)$, it follows from the local regularity of the outbranching in $\mathcal{T}$ and (15), that $\rho^{+}=\rho=1 / m$. 
We claim that

$$
\rho^{-}=\frac{1}{m} \sum_{k \geq 0}\left(\rho^{+}\left(1-\frac{1}{m}\right)\right)^{k}=\frac{m}{m^{2}-m+1} .
$$

Explanation: We have to consider a walk on $0,1, \ldots$, where the probability of going left is $1 / m$ at $i=1$ and $1 /(m+1)$ for $i>1$. Under these circumstances the summand represents the probability of $k$ returns to $i=1$ that then move right followed by a return to 0 .

Case (c): This is similar to the above case, except that for some vertices $v_{i}$ we remove one of their out-edges and make them absorbing. This makes a return to $\gamma$ less likely.

Case (d): Next consider the case of a unique light cycle $C$. Arguing as for Case (a) we have

$$
\rho \leq \frac{k-\delta_{k}}{k+l} \rho^{+}+\frac{l-\delta_{l}}{k+l} \rho^{-}+\frac{1}{k+l} .
$$

This is because only one of the edges $g$ leaving $\gamma(v)$ contains the vertex $x$ that loses an outedge, see property P3. For an upper bound we make the return probability 1 for going down this edge. Either $k$ or $l$ will be reduced by 1 .

The next step is to relate returns in $\mathcal{T}$ to returns in $\Gamma$. For most vertices an upper bound will suffice. For locally regular vertices we need a precise estimate.

Lemma 7. Let $r \leq \varepsilon \log \log n$, where $\varepsilon<1 / \log m$. Let $T=A \log n$ be given by (11). Then

(a) For all $v \in V$,

$$
R_{\gamma} \leq \frac{1+o(1)}{1-\rho}
$$

where the value of $\rho$ is given in Lemma 6

(b) If $v$ is locally regular then

$$
R_{\gamma}=\frac{m}{m-1}+O\left(\frac{1}{\log ^{\delta} n}\right)
$$

where $\delta>0$ constant.

\section{Proof Certainly}

$$
R_{\gamma}=\sum_{t=0}^{\omega} r_{t}+\sum_{t=\omega+1}^{T} r_{t} .
$$

Using (9) (with $x=u=\gamma$ ) we have

$$
\left|P_{\gamma}^{(t)}(\gamma)-\pi_{\gamma}\right| \leq e^{-t \Phi^{2} / 2},
$$

and thus for any $\omega$

$$
\begin{aligned}
\sum_{t=\omega+1}^{T} r_{t} & \leq T \pi_{\gamma}+\sum_{t=\omega+1}^{T} e^{-t \Phi^{2} / 2} \\
& =T \pi_{\gamma}+O\left(e^{-\omega \Phi^{2} / 2}\right),
\end{aligned}
$$


where $e^{-\Phi^{2} / 2}<1$ is constant follows from [12] and $G(m, n)$ nice.

We now compute an upper bound for $\sum_{t=0}^{\omega} r_{t}$. Let $\rho(H, t)$ denote the probability of return to $\gamma$ in graph $H$ at some step $s \leq t$. Assume first that $M_{\omega}^{\Gamma}(\gamma)$ contains no small cycles containing heavy vertices, so that $\mathcal{T}$ has no absorbing states at a finite distance from $\gamma$. Then from Raleigh's monotonicity law

$$
\rho(\Gamma, \omega) \leq p_{e s c}\left(\gamma, N_{\omega}^{\Gamma}(\gamma), \Gamma\right) \leq p_{e s c}\left(\gamma, N_{\omega}^{\Gamma}(\gamma), \mathcal{T}\right) \leq \rho(\mathcal{T})
$$

and hence

$$
\sum_{t=0}^{\omega} r_{t} \leq \frac{1}{1-\rho(\mathcal{T})}
$$

If there are small cycles with heavy vertices, then we made these vertices absorbing. The effect of this was to alter the above bound by $o\left(\omega^{-1}\right)$. Indeed, a random walk of length $\omega$ that starts at $\gamma$ might as well terminate if it reaches a vertex $w \leq n^{1 / 10}, w \neq \gamma$. By the properties assumed in Section 2.1 we have $d(w) \geq n^{1 / 4}$ (see P1), and that at least $n_{0}=n^{1 / 4}-(\log n)^{10 \omega}$ of the edges incident with $w$ are not in any cycle $C_{w}$ contained in $M_{\omega}(\gamma)$ (see P4). But then if a walk arrives at $w$, it has a more than $n_{0} / n^{1 / 4}$ chance of entering a sub-tree $T_{w}$ of $M_{\omega}(\gamma)$ rooted at $w$ for which every vertex is separated from $\gamma$ by $w$. The probability of leaving $T_{w}$ in $\omega$ steps is $O\left(\omega(\log n)^{10 \omega} / n^{1 / 4}\right)$ and so once a walk has reached $w$, the expected number of further returns to $\gamma$ is $o\left(\omega^{-1}\right)$.

(b) Locally regular vertices. We have $r_{t}=r_{t}(\gamma, \Gamma)=r_{t}(\gamma, \mathcal{T})$ for $t \leq \omega_{0}$. Thus

$$
\sum_{t=0}^{\omega} r_{t} \geq \frac{1}{1-\rho(\mathcal{T})}-\zeta
$$

where $\rho(\mathcal{T})=1 / m$ and $\zeta$ is the probability of a return to $\gamma$ in $\mathcal{T}$ at $t>\omega_{0}$.

For a walk on $(0,1,2, \ldots)$ with reflection at 0 , let $X_{t} \geq 0$ be the distance from the origin at step $t$. Couple this with $Z_{t}$ the distance from 0 for a walk on $(0, \pm 1, \pm 2, \ldots)$ with the same transition probabilities $p, q$. Clearly $\operatorname{Pr}\left(X_{t} \leq L\right) \leq \operatorname{Pr}\left(Z_{t} \leq L\right)$. Also

$$
\mathbf{E}\left(Z_{t}-Z_{t-1}\right)=p-q=\frac{m-1}{m+1} .
$$

Thus $\mathbf{E}\left(Z_{t}\right)=t(m-1) /(m+1)$. As

$$
\operatorname{Pr}\left(Z_{t} \leq 0\right) \leq \operatorname{Pr}\left(Z_{t} \leq \mathbf{E}\left(Z_{t}\right) / 2\right)=O\left(e^{\left.-\mathbf{E}\left(Z_{t}\right) / 8\right)}\right)
$$

then for some $\delta>0$ we have

$$
\zeta \leq \sum_{t \geq \omega_{0}} \operatorname{Pr}\left(Z_{t} \leq 0\right)=O\left(\frac{1}{\log ^{\delta} n}\right)
$$

Finally, after all this, we get what we need, i.e. the ratio $d(\gamma(v)) / R_{\gamma}$. 
Corollary 8. Let $1 \leq r \leq \varepsilon \log \log n$, where $\varepsilon<1 / \log m$. Then

(a) If $v$ is is locally tree-like then $d(\gamma(v)) / R_{\gamma} \geq m^{r}(m-1)(1-o(1))$.

(b) If $v$ is locally regular then $d(\gamma(v)) / R_{\gamma}=m^{r}(m-1)(1+o(1))$.

(c) If $v$ is is not locally tree-like then $d(\gamma(v)) / R_{\gamma} \geq m^{r+1}-m^{r}+m^{-1}-1$.

\section{Proof}

(a) Let $\rho=\rho_{\mathcal{T}}(k, l)$ be the first return probability given in Lemma 6 . Then

$$
\frac{d(\gamma(v))}{R_{\gamma}} \geq(k+l)(1-\rho)(1+o(1))
$$

However from (16)

$$
(k+l)(1-\rho)=k\left(1-\rho^{+}\right)+l\left(1-\rho^{-}\right) \geq k\left(1-\rho^{+}\right) .
$$

Since $v$ is tree-like, $k \geq m^{r+1}$ and $\rho^{+}=1 / m$, giving part (a).

(b) When $v$ is locally regular, we have $k=m^{r+1}$ and $l=0$ and we can apply the above analysis.

(c) Our first task is to find lower bounds on the values of $k, l$ for these vertices. Suppose first that $M_{r}^{G}(v)$ contains a vertex $w \leq n^{1 / 10}$. If $\left|M_{r}^{G}(v)\right| \leq n^{1 / 4} / 2$ then (3) implies that $k+l \geq$ $n^{1 / 4} / 2$. Otherwise, Property P8 implies that $\left|M_{r}^{G}(v)\right| \geq n^{1 / 4} / 2$ and $\sum_{x \in M_{r}^{G}(v)} d(x) \leq n^{9 / 10}$. But then Property P7 implies that $k+l \geq m n^{1 / 4} \Phi / 2$. In summary, if $M_{r}^{G}(v)$ contains a heavy vertex then $k+l \geq c_{1} n^{1 / 4}$ for some constant $c_{1}>0$. From Lemma 6 we then have

$$
\frac{d(\gamma(v))}{R_{\gamma}(1-o(1))} \geq(k+l)(1-\rho) \geq k+l-\frac{k-\delta_{k}}{m}-\frac{\left(l-\delta_{l}\right) m}{m^{2}-m+1}-1 \geq \frac{c_{1} n^{1 / 4}}{4} \gg m^{r+1} .
$$

Suppose next that $M_{r}^{G}(v)$ contains a light cycle $C$. Property P3 implies that this can be the only cycle in $M_{r+\omega}^{G}(v)$. Because a triangle incident with $v$ has the most effect in reducing $k$ we deduce from this that

$$
k \geq(m-1)\left(m^{r}+m^{r-1}\right)
$$

and

$$
\frac{d(\gamma(v))}{R_{\gamma}(1-o(1))} \geq(m-1)\left(m^{r}+m^{r-1}\right)(1-\rho)=(m-1)^{2}(m+1) m^{r-2} .
$$

We are now left with the case where $M_{r}^{G}(v)$ is cycle free but $M_{r+\omega}^{G}(v)$ is not. If $M_{r+\omega}^{G}(v)$ contains a heavy vertex then Lemma 6(c) implies that we can use the lower bound of part 
(a). Otherwise, $M_{r+\omega}^{G}(v)$ contains a unique light cycle and from Lemma $6(\mathrm{~d})$ we have

$$
\begin{aligned}
\frac{d(\gamma(v))}{R_{\gamma}(1-o(1))} & \geq k+l-\frac{k-\delta_{k}}{m}-\frac{\left(l-\delta_{l}\right) m}{m^{2}-m+1}-1 \\
& \geq m^{r+1}-\frac{m^{r+1}-1}{m}-1 .
\end{aligned}
$$

\subsection{Verifying the conditions of Lemma 5}

Lemma 5 uses the condition that $T \pi_{\gamma(v)}=o(1)$, so we only use this lemma for vertices of degree at most $D=2 T m^{r+1}$. Vertices $v$, such that $d(\gamma(v)) \geq D$ are dealt with in an ad-hoc fashion (see Lemma 10 of Section 5).

Lemma 9. There exists a constant $0<\theta<1$ such that if $v \in V$ then $\left|R_{T}(z)\right| \geq \theta$ for $|z| \leq 1+\lambda$.

Proof The proof is similar to that given in Lemma 16 of [7], but we give it here for completeness.

Assume first that $v$ is locally tree-like. Let $\mathcal{T}_{1}$ be the infinite tree obtained by attaching disjoint copies of the infinite tree $T_{m}^{\infty}$ with branching factor $m$ to all leaves of $M_{\omega}^{\Gamma}(\gamma(v))$ at depth $\omega$. Thus $\mathcal{T}_{1}$ is $\mathcal{T}$ without the edge deletions. We write

$$
\begin{aligned}
R_{T}(s) & =A(s)+Q(s) \\
& =\frac{1}{1-B(s)}+Q(s) .
\end{aligned}
$$

Let $\mathcal{W}_{\gamma(v)}^{*}$ denote a random walk on the tree $\mathcal{T}_{1}$. Then $A(s)=\sum a_{t} s^{t}$ where $a_{t}=r_{t}^{*}$ is the probability that the random walk $\mathcal{W}_{\gamma(v)}^{*}$ is at $\gamma(v)$ at time $t . B(s)=\sum b_{t} s^{t}$ where $b_{t}$ is the probability of a first return at time $t$ for the random walk $\mathcal{W}_{\gamma(v)}^{*}$. Then $Q(s)=Q_{1}(s)+Q_{2}(s)$ where

$$
\begin{aligned}
& Q_{1}(s)=\sum_{t=\omega+1}^{T}\left(r_{t}-a_{t}\right) s^{t} \\
& Q_{2}(s)=-\sum_{t=T+1}^{\infty} a_{t} s^{t} .
\end{aligned}
$$

Here we have used the fact that $a_{t}=r_{t}$ for $0 \leq t \leq \omega$.

We now justify equation (21). For this we need to show that

$$
|B(s)|<1 \quad \text { for }|s| \leq 1+\lambda .
$$


We note first that, in the notation of Lemma $6, B(1) \leq \rho(k, l)<1$. Then observe that $b_{t} \leq a_{t} \leq e^{-\alpha t}$. The latter inequality can be proved following the same arguments as given in the proof of (42) in [7]. Thus the radius of convergence $\rho_{B}$ of $B(s)$ is at least $e^{\alpha}, B(s)$ is continuous for $0 \leq|s|<\rho_{B},|B(s)| \leq B(|s|)$ and $B(1)<1$. Thus there exists a constant $\epsilon>0$ such that $B(s)<1$ for $|s| \leq 1+\epsilon$. We can assume that $\lambda<\epsilon$ and (22) follows. We will use

$$
\left|R_{T}(s)\right| \geq \frac{1}{1+B(|s|)}-|Q(s)| \geq \frac{1}{1+B(1+\lambda)}-|Q(s)| \geq \frac{1}{2}-|Q(s)| .
$$

The lemma for locally tree-like vertices will follow once we show that $|Q(s)|=o(1)$. But, using (9) (with $x=u=\gamma$ ),

$$
\begin{aligned}
& \left|Q_{1}(s)\right| \leq(1+\lambda)^{T} \sum_{t=\omega+1}^{T}\left(\pi_{v}+e^{-\Phi^{2} t / 2}+e^{-\alpha t}\right)=o(1) \\
& \left|Q_{2}(s)\right| \leq \sum_{t=T+1}^{\infty}\left(e^{-\alpha}(1+\lambda)\right)^{t}=o(1) .
\end{aligned}
$$

Suppose next that $v$ is not locally tree-like. Assume first that $M_{\omega}^{\Gamma}(\gamma)$ only contains cycles with heavy vertices. We truncate $M_{\omega}^{\Gamma}(\gamma)$ at vertices of degree more than $n^{1 / 4}$, add copies of $T_{m}^{\infty}$ at leaves as before and then proceed as above. The point is that the truncation can only change $r_{t}, t \leq T$ by $O\left(T n^{-1 / 4}\right)$.

Finally, consider the case where $M_{\omega}^{\Gamma}(\gamma)$ contains a unique light cycle $C=\left(x_{1}, x_{2}, \ldots, x_{k}, x_{1}\right)$. We can add copies of $T_{m}^{\infty}$ at leaves as before and write an expression equivalent to (21) and then the argument rests on showing that $B(1)<1$ and $a_{s} \leq \zeta^{s}$ for some $\zeta<1$. The latter condition can be relaxed to $a_{s} \leq e^{o(s)} \zeta^{s}$, allowing us to take less care with small $s$.

$\boldsymbol{B}(\mathbf{1})<1$ : We can assume that $r \geq 1$, since the case $r=0$ is the subject of [7]. If $k, l$ are as in Lemma 6 then whp we have $k+l \geq 3$ and then there is a $\geq 1-\frac{2}{k+l}$ probability of the first move of $\mathcal{W}_{v}^{*}$ going into an infinite tree rooted at a neighbour of $\gamma(v)$ and then the probability of return to $\gamma(v)$ is bounded below by a positive constant.

$\boldsymbol{a}_{\boldsymbol{s}} \leq \boldsymbol{e}^{\boldsymbol{o}(s)} \boldsymbol{\zeta}^{s}$ : We can couple the distance $X_{t}$ of $\mathcal{W}_{v}^{*}(t)$ to $\gamma(v)$ with a random walk on $\{0,1,2, \ldots$,$\} . In all cases we find that \mathbf{E}\left(X_{t+2}-X_{t}\right) \geq 0$ and $\mathbf{E}\left(X_{t+4}-X_{t}\right)$ is strictly positive and we can use Hoeffding's theorem.

\section{Upper bound on cover time}

We now consider the upper bound in Theorem 2. Let $t^{*}=\frac{2}{m^{r-1}(m-1)} n \log n$, and let $t_{1}=$ $t^{*}(1+\epsilon)$, where $\epsilon \rightarrow 0$ sufficiently slowly, so that any subsequently claimed inequalities are valid. An upper bound of $t_{1}$ for the cover time is established below in Lemmas 10 and 11. 
Our basic approach is as follows. Let $T_{G}(u)$ be the time taken for the random walk $\mathcal{W}_{u}$ to visit every vertex of a connected graph $G$. Let $U_{t}$ be the number of vertices of $G$ which have not been visited by $\mathcal{W}_{u}$ at step $t$. Then

$$
\begin{aligned}
C_{u}=\mathbf{E}\left(T_{G}(u)\right) & =\sum_{t>0} \operatorname{Pr}\left(T_{G}(u) \geq t\right), \\
\operatorname{Pr}\left(T_{G}(u) \geq t\right)=\operatorname{Pr}\left(T_{G}(u)>t-1\right) & =\operatorname{Pr}\left(U_{t-1}>0\right) \leq \min \left\{1, \mathbf{E}\left(U_{t-1}\right)\right\} .
\end{aligned}
$$

We first deal with vertices $v$ with $d(\gamma(v))$ greater than $D$.

Lemma 10. Let $D=2 T m^{r+1}$, let $V_{L}=\{v: d(\gamma(v)) \geq D\}$. Then $t_{1}$ is an upper bound on the expected time to cover $V_{L}$.

Proof Let $\tau_{v}(u)$ be the time taken for $\mathcal{W}_{u}$ to visit $v \in V_{L}$. Then by considering $\mathcal{W}_{u}(T), \mathcal{W}_{u}(2 T) \ldots$ we see that

$$
\begin{aligned}
\sum_{t \geq t_{1}} \operatorname{Pr}\left(\tau_{v}(u) \geq t\right) & \leq \sum_{j \geq\left\lceil t_{1} / T\right\rceil}\left(1-\frac{D(1+o(1))}{2 m n}\right)^{j} \\
& =O\left(\exp \left(-(1+o(1)) \frac{D t_{1}}{2 m n T}\right)\right) \\
& =O\left(n^{-1}\right) .
\end{aligned}
$$

Lemma 11. Let $V^{\prime}=V \backslash V_{L}$. Then $t_{1}$ is an upper bound on the expected time to cover $V^{\prime}$.

Proof Recall that $\boldsymbol{A}_{t}(v), t \geq T$ is the event that $\mathcal{W}_{u}$ does not visit $v$ at steps $T, T+$ $1, \ldots, t$. Then

$$
C_{u} \leq t+1+\sum_{s \geq t} \mathbf{E}\left(U_{s}\right) \leq t+1+\sum_{v} \sum_{s \geq t} \operatorname{Pr}\left(\boldsymbol{A}_{s}(v)\right) .
$$

Fix $u \in V$ and let $C_{u}(r)$ be the expected time for $\mathcal{W}_{u}$ to have been within distance $r$ of every vertex. It follows from (23), (24) that for all $t \geq T$,

$$
C_{u}(r) \leq t+1+\sum_{v \in V} \sum_{s \geq t} \operatorname{Pr}\left(\boldsymbol{A}_{s}^{(r)}(v, G)\right)
$$

where $\boldsymbol{A}_{s}^{(r)}(v, G)$ is the event that the walk $\mathcal{W}_{u}$ in $G$, has not been within distance $r$ of $v$ in the interval $[T, t]$.

Let $\gamma(v)$ be the contraction of $M_{r}(v)$ as described in the construction at the start of Section 4.3. We note the following:

- If $w \notin M_{r}^{G}(v)$, then $\pi_{w}(G)=\pi_{w}(\Gamma)(1+O(d(\gamma) / n))$. 
- Thus $\operatorname{Pr}\left(\mathcal{W}_{u}^{G}(T)=w\right)=\operatorname{Pr}\left(\mathcal{W}_{u}^{\Gamma}(T)=w\right)(1+O(d(\gamma) / n))$.

- We can couple a random walk in $G$ starting at $w \notin M_{r}(v)$ with a random walk in $\Gamma$ starting at $w$ up until the second walk visits $\gamma(v)$ in a measure preserving way.

Thus

$$
\operatorname{Pr}\left(\boldsymbol{A}_{s}^{(r)}(v, G)\right) \sim \operatorname{Pr}\left(\boldsymbol{A}_{s}(\gamma(v), \Gamma)\right),
$$

where $\operatorname{Pr}\left(\boldsymbol{A}_{s}(\gamma(v), \Gamma)\right)$ is given by Lemma 5 , on choosing $v:=\gamma(v)$ in (8). The precise value of $p_{\gamma}$ for $\gamma(v)$ in $(7)$ is given $d(\gamma) /\left(R_{\gamma} d(\Gamma)\right)$, where $d(\gamma) / R_{\gamma}$ is given by Corollary 8 , and $d(\Gamma)=2 m n(1-O(d(\gamma))$.

Partition $V^{\prime}$ into $V_{T}, V_{C}$ the vertices with tree-like and non-tree-like neighbourhoods of $\gamma$. For tree-like neighbourhoods,

$$
\begin{aligned}
\sum_{v \in V_{T}} \sum_{s \geq t_{1}} \operatorname{Pr}\left(\boldsymbol{A}_{s}(\gamma(v))\right) & \leq(1+o(1)) n \sum_{s \geq t_{1}} e^{-s p_{\gamma}(v)} \\
& \leq 2 n^{2} e^{-(1+\epsilon / 2) t^{*} m^{r}(m-1) /(2 m n)} \\
& =O\left(n^{2} n^{-(1+\epsilon / 2)}\right) \\
& =o\left(t_{1}\right) .
\end{aligned}
$$

For non-tree-like neighbourhoods, using property P6 we see that

$$
\begin{aligned}
\sum_{v \in V_{T}} \sum_{s \geq t_{1}} \operatorname{Pr}\left(\boldsymbol{A}_{s}(\gamma(v))\right) & \leq O\left(n^{1 / 2+o(1)}\right) \sum_{s \geq t_{1}} e^{-s p_{\gamma}(v)} \\
& \leq 2 n^{3 / 2+o(1)} e^{-(1+\epsilon / 2) t^{*}\left(m^{r+1}-m^{r}+m^{-1}-1\right) /(2 m n)} \\
& =O\left(n^{3 / 2+o(1)} n^{-(1+\epsilon / 2)\left(1-(m-1) / m^{r+1}\right)}\right) \\
& =o\left(t_{1}\right) .
\end{aligned}
$$

Note that we assume $r \geq 1$ to get the final expression. The case $r=0$ is the content of [7].

\section{Lower bound on cover time}

Lemma 12. If $G$ is nice then there is a set $S$ of locally regular vertices, $|S|=n^{1-o(1)}$, such that if $v, w \in S$ then the distance between any vertex of $M_{r}(\gamma(v))$ and $M_{r}(\gamma(w))$ is at least $10 \omega$.

Proof Let $S_{1}$ be the set of locally regular vertices described in property P2. They satisfy property P1, and have at most $(\log n)^{100 \omega}$ neighbours at depth $20 \omega$ by property P5. Then $\left|S_{1}\right|=n^{1-o(1)}$ and there is a subset $S$ of $S_{1}$ of size at least $\left|S_{1}\right| /(\log n)^{100 \omega}$ satisfying the requirements of the lemma. 
Let $t_{0}=t_{1}^{*}(1-\epsilon)$ where $\epsilon \rightarrow 0$ sufficiently slowly that any subsequently claimed inequalities are valid. Choose $u \in S$. We prove that at time $t_{0}$, the probability that the set $S$ is covered by the walk $\mathcal{W}_{u}$ tends to zero.

For each pair of vertices $v, w \in S \backslash\{u\}$ form $\Gamma(v, w)$ by contracting $M_{r}\left(v_{1}\right) \cup M_{r}\left(v_{2}\right)$ into a single vertex $\gamma(v, w)$ and removing loops. Note that the mixing time $T_{\Gamma(v, w)} \leq T_{\Gamma(v)}+T_{\Gamma(w)}$.

Lemma 13. Let $v, w \in S$. Then

$$
R_{\gamma(v, w)}=\frac{1}{2} R_{\gamma(v)}+\frac{1}{2} R_{\gamma(w)}+o\left(\frac{1}{\log n}\right) .
$$

Proof The probability $\rho^{*}$ of a first return to $\gamma(v, w)$ during $T_{\Gamma(v, w)}$ is given by

$$
\rho^{*}=\frac{1}{2}\left(\rho_{\gamma(v)}+\rho_{\gamma(w)}\right)+\zeta(v, w)
$$

where $\zeta(v, w)$ is the probability of a first passage between $\gamma(v)$ and $\gamma(w)$ during $T_{\Gamma(v, w)}$ in the graph formed by contracting $M_{r}(v)$ to $\gamma(v)$ and $M_{r}(w)$ to $\gamma(w)$. Let $\gamma=\gamma(v, w)$. This passage requires at least $7 \omega$ steps of the walk, and we have (see $(17)$ ) for $7 \omega<t \leq T_{\Gamma(v, w)}$ that

$$
\left|P_{\gamma}^{(t)}(\gamma)-\pi_{\gamma}\right| \leq e^{-\Phi^{2} t / 2}
$$

Thus for some constant $A>1$,

$$
\zeta(v, w)=T_{\Gamma(v, w)} O\left(\pi_{\gamma}+A^{-\omega}\right)=o\left(1 / \log ^{k} n\right),
$$

for any integer $k$.

By the same token the expected number of returns to $v$ between $T_{\Gamma(v)}$ and $T_{\Gamma(v)}+T_{\Gamma(w)}$ is also $o\left(1 / \log ^{k} n\right)$.

Hence $T_{1}(u)>t_{0}$ whp which implies that $C_{G} \geq t_{1}^{*}-o\left(t_{1}^{*}\right)$.

Let $Y_{v}$ be the indicator for the event $\mathcal{A}_{t_{0}}^{(r)}(v)$. Let $X=\sum_{v \in S} Y_{v}$ denote the subset of $S$ which is unvisited in $\left[T, t_{0}\right]$. It follows from $(8)$ that

$$
\mathbf{E}(X)=\sum_{v \in S} \operatorname{Pr}\left(\boldsymbol{A}_{t_{0}}^{(r)}(v)\right)=\sum_{v}\left(1+O\left(T \pi_{v}\right)\right) e^{-t_{0} p_{v}}+o\left(\sqrt{n} e^{-\lambda t_{0} / 2}\right)=n^{1-o(1)} .
$$

Having bounded $\mathbf{E}(X)$ from below we continue by estimating the second moment of $X$.

Fix $v, w \in S$. We will show that

$$
\operatorname{Pr}\left(\mathcal{A}_{t_{0}}^{(r)}(v) \wedge \mathcal{A}_{t_{0}}^{(r)}(w)\right)=(1+o(1)) \operatorname{Pr}\left(\mathcal{A}_{t_{0}}^{(r)}(v)\right) \operatorname{Pr}\left(\mathcal{A}_{t_{0}}^{(r)}(w)\right)
$$

It then follows that

$$
\mathbf{E}\left(X^{2}\right)=(1+o(1)) \mathbf{E}(X)^{2}
$$


Using the Chebyshev inequality we see that

$$
\operatorname{Pr}(X<\mathbf{E}(X / 2))=o(1)
$$

and thus whp at least $\mathbf{E}(X) / 2-T>0$ vertices of $S$ are unvisited at $t_{0}$.

So, with $\mathbf{P r}^{*}$ referring to probability in the space of random walks on $\Gamma(v, w)$, and using Lemma 13 and $R_{\gamma(v)} \sim R_{\gamma(w)}$,

$$
\begin{aligned}
\operatorname{Pr}^{*}\left(\mathcal{A}_{t_{0}}(\gamma(v, w))\right) & =(1+o(1)) \exp \left\{-\frac{t_{0} \pi_{\gamma(v, w)}}{\left(1+O\left(T \pi_{\gamma(v, w)}\right) R_{\gamma(v, w)}\right.}\right\} \\
& =(1+o(1)) \exp \left\{-\frac{t_{0} \pi_{\gamma(v)}}{R_{\gamma(v)}}\right\} \exp \left\{-\frac{t_{0} \pi_{\gamma(w)}}{R_{\gamma(w)}}\right\} \\
& =(1+o(1)) \operatorname{Pr}\left(\mathcal{A}_{t_{0}}(\gamma(v))\right) \operatorname{Pr}\left(\mathcal{A}_{t_{0}}(\gamma(w))\right) \\
& =(1+o(1)) \operatorname{Pr}\left(\mathcal{A}_{t_{0}}^{(r)}(v)\right) \operatorname{Pr}\left(\mathcal{A}_{t_{0}}^{(r)}(w)\right)
\end{aligned}
$$

But, using rapid mixing in $\Gamma(v, w)$,

$$
\begin{aligned}
\operatorname{Pr}^{*}\left(\mathcal{A}_{t_{0}}(\gamma(v, w))\right) \\
=\sum_{x \neq \gamma(v, w)} P_{u}^{T_{\Gamma(v, w)}}(x) \operatorname{Pr}^{*}\left(\mathcal{W}_{x}\left(t-T_{\Gamma(v, w)}\right) \neq \gamma(v, w), T_{\Gamma(v, w)} \leq t \leq t_{0}\right) \\
=\sum_{x \neq \gamma(v, w)}\left(\frac{d e g(x)}{2 m n}+O\left(n^{-2+o(1)}\right)\right) \operatorname{Pr}^{*}\left(\mathcal{W}_{x}\left(t-T_{\Gamma(v, w)}\right) \neq \gamma(v, w), T_{\Gamma(v, w)} \leq t \leq t_{0}\right) \\
=\sum_{x \neq v, w}\left(P_{u}^{T_{\Gamma(v, w)}}(x)+O\left(n^{-2+o(1)}\right)\right) \operatorname{Pr}\left(\mathcal{W}_{x}\left(t-T_{\Gamma(v, w)} \notin M_{r}^{G}(v) \cup M_{r}^{G}(w), T_{\Gamma(v, w)} \leq t \leq t_{0}\right)\right. \\
=\operatorname{Pr}\left(\mathcal{W}_{u}(t) \notin M_{r}^{G}(v) \cup M_{r}^{G}(w), T_{\Gamma(v, w)} \leq t \leq t_{0}\right)+O\left(n^{-2+o(1)}\right) \\
=\operatorname{Pr}\left(\mathcal{A}_{t_{0}}^{(r)}(v) \wedge \mathcal{A}_{t_{0}}^{(r)}(w)\right)+O\left(n^{-2+o(1)}\right) .
\end{aligned}
$$

Equation (32) follows because there is a natural measure preserving map $\phi$ between walks in $G$ that start at $x \neq v, w$ and avoid $M_{r}^{G}(v) \cup M_{r}^{G}(w)$ and walks in $\Gamma(v, w)$ that avoid $\gamma(v, w)$.

This completes the proof of Theorem 2.

\section{References}

[1] A. Barabási and R. Albert, Emergence of scaling in random networks, Science 286 (1999) 509-512.

[2] B. Bollobás and O. Riordan, The diameter of a scale-free random graph, Combinatorica 24 (2004) 5-34. 
[3] A. Broder, R. Kumar, F.Maghoul, P. Raghavan, S. Rajagopalan, R. Stata, A. Tomkins and J. Wiener, Graph structure in the web, Proc. 9th WWW Conf. 309-320 (2000).

[4] C. Cooper The age specific degree distribution of web-graphs. Combinatorics Probability and Computing 15(5), 637-661, (2006).

[5] C. Cooper, and A. M. Frieze. A general model of web graphs. Random Structures and Algorithms, 22, 311-335 (2003).

[6] C. Cooper and A. M. Frieze, The cover time of random regular graphs, SIAM Journal on Discrete Mathematics, 18 (2005) 728-740.

[7] C. Cooper and A. M. Frieze, The cover time of the preferential attachment graph, Journal of Combinatorial Theory Series B, 97 (2007) 269-290.

[8] C. Cooper and A. M. Frieze. The cover time of the giant component of $G_{n, p}$. Random Structures and Algorithms 32, 401-439 J. Wiley (2008).

[9] P. G. Doyle and J. L. Snell, Random Walks and Electrical Networks. Carus Mathematical Monograph 22, AMA (1984).

[10] M. Faloutsos and P. Faloutsos and C. Faloutsos, On Power-law Relationships of the Internet Topology, SIGCOMM 251-262 (1999).

[11] W. Feller, An Introduction to Probability Theory and Its Applications, Volume 1, Wiley and Sons, New York, 1971.

[12] M. Mihail, C. Papadimitriou, and A. Saberi , On Certain Connectivity Properties of the Internet Topology, Proceedings of the 44th Symposium on Foundations of Computer Science (2003) 28-35.

[13] M. Mihail, A. Saberi, and P. Tetali, Random Walks with Lookahead in Power Law Random Graphs. Internet Mathematics 3(2) (2007).

[14] A. Sinclair. Improved bounds for mixing rates of Markov chains and multicommodity flow. Combinatorics, Probability and Computing, 1 (1992), 351-370. 